\title{
Aproximación matemática al proceso de galvanorrecocido en productos siderúrgicos planos
}

\author{
A. Pérez ${ }^{(*)}$, I. González ${ }^{(* *)}$, L.J. Andrés ${ }^{(*)}$, B. Fernández ${ }^{(* *)}$ y J.M. Puente $\left(^{(* *)}\right.$
}

Resumen Se revisan brevemente los modelos cinéticos descritos para la formación de las fases de aleación ZnFe durante el proceso de galvanorrecocido de chapa, con especial énfasis en el proceso continuo, con destino a la industria del automóvil. Se ha realizado una primera aproximación matemática al crecimiento cinético de la fase delta utilizando un simulador de recubrimiento en continuo por inmersión en caliente produciéndose el galvanorrecocido por calentamiento de la chapa galvanizada exactamente después de su salida del baño de zinc con objeto de aproximarse a las condiciones reales imperantes en las líneas de producción. Los parámetro variables han sido: el tiempo y la temperatura de galvanorrecocido y el peso de recubrimiento. Lost resultados iniciales del contenido de hierro del recubrimiento y de contenido de fase delta están de acuerdo con los resultados obtenidos por otros autores..

Palabras clave: Galvanorrecocido. Simulador. Modelos cinéticos. Aleaciones de zinc.

\section{Mathematical approach in galvannealed steel sheet coatings}

\begin{abstract}
A short review of the kinetics models for the formation of Fe-Zn alloy phases in the galvannealing process is presented. It will focus on the continuous process which is often used by the automotive industry. A first mathematical approach of the kinetics growth of the $\delta$ phase has been done, using a continuous hot-diping process simulator which resembles the conditions of the galvannealing process in production lines. Hold time and the galvannealing temperature as well as the weight of the coating were varied. The preliminary results of the iron content and proportion of $\delta$ phase in the coating are in agreement with the results obtained by other authors.
\end{abstract}

Keywords: Hot-dip simulator. Galvannealing. Mathematical models. Zinc alloys.

\section{INTRODUCCIÓN}

El galvanorrecocido es el resultado de la aleación del zinc con el acero base en un recubierto galvanizado. El tratamiento térmico genera la aparición de varias fases, cuyo contenido de hierro, estructura y proporción relativa dependen en gran medida de la historia térmica del tratamiento y de su duración. Otros factores que se han revelado cruciales son la calidad del acero base y la composición y naturaleza de los aditivos del pote de inmersión.

(*) Instituto Tecnológico de Materiales. TMA. Parque Tecnológico de Asturias. 33428- Coruño - Llanera (Asturias, España). Fax: +34/8/5265574

(**) ACERALIA Corp. Siderúrgica. Centro de Desarrollo Tecnológico. Aptdo. 90.33480- Avilés (Asturias, Espana). Fax: $+34 / 8 / 5126089$
Se sabe que el tipo, espesor y morfología de estas fases influyen tanto en su resistencia a la corrosión como en sus propiedades mecánicas a la hora de la conformación, por lo que un adecuado diseño de estas propiedades permite su amplia utilización en la industria del automóvil. Son fundamentales la forma de conducir el estudio y la idoneidad del equipo utilizado.

Dado el elevado número de parámetros que influyen en el diseño final de la estructura y, por tanto, de las propiedades finales del galvanorrecocido, se ha realizado un gran esfuerzo investigador, desde su aparición, para determinar los mecanismos de formación de dichas fases así como de sus propiedades desde los puntos de vista mecanicistas y fenomenológicos que permiten, en algunos casos, obtener ecuaciones útiles en la estimación de las 
condiciones de fabricación de estos recubiertos con vistas a una determinada aplicación final.

En el presente trabajo se expone de forma breve el estado del tema, de los equipos de simulación y ensayo disponibles y de algunos resultados iniciales al abordar la aproximación matemática al proceso de galvanorrecocido.

\section{MODELOS}

De los aditivos utilizados en el pote de inmersión, especial atención ha tenido el aluminio por su capacidad para retardar la reacción inicial, no homogénea, entre el sustrato de acero y el zinc del baño a través de la formación de una capa de $\mathrm{Fe}_{2} \mathrm{Al}_{5}-\mathrm{Zn}_{\mathrm{x}}$, proponiéndose un modelo de formación y crecimiento de esta fase ( 1 y 2 ). Estos mismos autores establecen que el contenido mínimo para una inhibición completa es de $0,15 \%$ a $450{ }^{\circ} \mathrm{C}$ aproximadamente, lo que está de acuerdo con otros resultados (3). El espesor de la capa intermetálica depende, fundamentalmente, de la concentración de aluminio en el pote y de la diferencia de temperatura a la entrada del pote (4).

En los últimos años, se ha comenzado a estudiar otros aditivos, manganeso (5), cobre (6) y titanio, siendo de destacar que la mayoría de estos estudios se han realizado en simuladores de recubrimiento en continuo como el utilizado en nuestro laboratorio, que permite simular las operaciones en continuo y en condiciones muy próximas a las reales de planta y no en etapas separadas (recocido, galvanizado y aleación galvanorrecocido) para evitar dificultades añadidas.

Superado un cierto tiempo de inhibición, dependiente de la naturaleza y estado superficial del sustrato (3), tiene lugar la difusión del hierro hacia el recubrimiento de zinc y el comienzo de la reacción de nucleación y crecimiento de fases. Estos mecanismos han sido modelizados fenomenológicamente, sobre todo para aceros $\mathrm{IF}(\mathrm{Ti})$, por varios autores (2, 7 y 8). La secuencia de desarrollo del galvanne$a l$, de acuerdo con varios autores, sería la que se describe en los apartados siguientes.

\subsection{Difusión del hierro a través de la intercara acero/recubrimiento}

Durante estos primeros instantes, el flujo difusional de hierro genera la aparición de estados incipientes de fase interfacial $\Gamma, \delta$ y $\zeta$ quedando en la superficie del recubrimiento algo de fase $\eta$ (zinc puro) sin transformar. La fase $\zeta$ aparece como cristales gruesos en el frente de difusión en contacto con la fase $\eta$, lo que sugiere que ésta se produce por nucleación sobre la fase $\eta$ debido a sobresaturaciones locales consumiendo en la transformación parte de la fase $\eta$.

\subsection{Progresión de la difusión del hierro}

En esta etapa, se produce un crecimiento rápido de la capa interfacial $\Gamma$ hasta un determinado espesor (aprox. $1 \mu \mathrm{m}$ ), permaneciendo estable. La fase $\delta$ crece por aporte difusional a expensas de la capa $\zeta$ en dirección a la superficie del recubrimiento. La fase $\zeta$ puede seguir generándose debido a la sobresaturación de la capa $\eta$. A tiempos suficientemente largos, la fase $\eta$ desaparece completamente y la $\zeta$ puede llegar a desaparecer o permanecer restos testimoniales en la superficie del recubrimiento.

\subsection{Inicio de la sobresaturación de la capa delta}

El enriquecimiento de la capa $\delta$ aumenta hasta un $12 \%$ aproximadamente (límite de solubilidad) si bien se puede sobrepasar este valor sin que se pueda justificar este comportamiento. La fase $\Gamma$ comienza nuevamente a crecer a costa de la $\delta$. Algunos cristales de fase $\zeta$ pueden permanecer en la superficie del recubrimiento. También se ha estudiado la cinética del crecimiento y morfología de una subcapa de la $\Gamma$, denominada $\Gamma 1$ (9).

La composición del acero base tiene un efecto fundamental sobre el grado de aleación del galvanorrecocido, determinándose que la velocidad de aleado aumenta en la secuencia (10):

Acero refosforado $<$ Acero calmado con $\mathrm{Al}<\mathrm{IF}$ (Ti)

Sin embargo, parece que la secuencia de generación y crecimiento de fases resulta independiente del acero base.

Se ha de hacer notar la dificultad del análisis de las aleaciones obtenidas, estando perfectamente establecida la necesidad de utilizar una combinación de técnicas analíticas para la determinación y valoración cuantitativa de sus propiedades (peso y espesor, composición, morfología, fases cristalinas). Las técnicas que se consideran mínimas son, análisis químico ICP, SEM con microanálisis EDAX, DRX y electrodisolución anódica selectiva, además de la capacidad de realización de ensayos mecánicos no convencionales (11). La preparación de las muestras a escala de laboratorio también presenta exigencias concretas si se quiere que sean representativas de las obtenidas en líneas continuas industriales. Para evitar incertidumbre y discrepancias con la realidad industrial, la simulación de las etapas de recocido, galvanizado y aleado deben realizarse en simuladores de forma continua (12). Se 
ha puesto de manifiesto que incluso en simuladores de este tipo el equipamiento debe ser el adecuado, debiendo utilizarse hornos de inducción para el proceso de galvanorrecocido y no de radiación-convección, como los utilizados habitualmente, si se quiere obtener resultados precisos (12); en particular, influye sobre la cinética de la aleación, ralentizándola. Son también deseables refinamientos como el control del punto de rocío de la atmósfera durante la operación.

Todo este equipamiento se encuentra disponible en nuestros laboratorios y se ha utilizado para el estudio del proceso de galvanorrecocido en nuevos aceros de alta resistencia con destino al sector de automoción.

\section{MODELOS MATEMÁTICOS}

En los último tiempos, se han realizado varias aproximaciones matemáticas (13-15) con el propósito de describir, total o parcialmente el modelo fenomenológico anteriormente descrito (o alguna de sus variantes). La parcialidad e imperfección de nuestros conocimientos actuales, la complejidad intrínseca del proceso, la multitud de variables implicadas y la falta de justificación para algunos fenómenos son las principales causas de la escasez de estos modelos y de su limitada capacidad para ajustarse a los datos experimentales.

Las simplificaciones más habituales introducidas en estos modelos son:

- La formación de las fases es secuencial siguiendo el orden zeta - delta - gamma

- La etapa controlante es un fenómeno de difusión (bien del zinc o del hierro) caracterizándose cada fase por un coeficiente de difusión propio y de valor constante.

- La composición del pote y la naturaleza del acero base sólo afectan al instante de comienzo de la fase (tiempo de retraso) y al valor de las constantes cinéticas encontradas.

- La temperatura se parametriza mediante la variación de las constantes cinéticas, correlacionándose a través de ecuaciones de tipo Arrhenius.

En algunos casos, se establecen ecuaciones cinéticas de primer orden respecto de un valor límite en el contenido de hierro de la fase $\delta$ (16). En estas ecuaciones, aparece frecuentemente el parámetro

$x / \sqrt{D t}$, y la función error complementaria $(e r f c)$ como corresponde a un proceso difusional por caminos paralelos, siendo necesario, en muchos casos, la resolución mediante métodos numéricos.

\section{SIMULADOR DE RECUBRIMIENTO EN CONTINUO POR INMERSIÓN EN CALIENTE}

Las figuras $1 a$ y $1 b$ muestran, respectivamente, un esquema del equipamiento del simulador utilizado y una vista global del mismo. Se trata de un simulador para procesos de recubrimiento en continuo por inmersión en caliente, que permite reproducir todas las etapas de tratamiento a partir de chapa laminada en frío: recocido en continuo, inmersión en el baño fundido, control de espesor del recubrimiento por cuchillas de soplado, aleado de la capa de recubrimiento y enfriamiento controlado. Puede utilizarse para la simulación de recubrimientos basados en HDG (Hot-Dip Galvanizing), Galfan (Zn/Al 95:5), Algafort (55\% Al, 43,4 \% Zn, 1,6\% $\mathrm{Si}$ ), Galvanneal (aleación de zinc con $10 \% \mathrm{Fe}$ ), Aluminizado tipo 1 y 2 , y permite la investigación de la influencia de aleantes minoritarios o aditivos en el baño fundido sobre estos recubrimientos.

Un ordenador permite establecer las velocidades de transferencia por cada etapa, la velocidad de calentamiento y enfriamiento, el tiempo de tratamiento y la composición y tipo de atmósfera
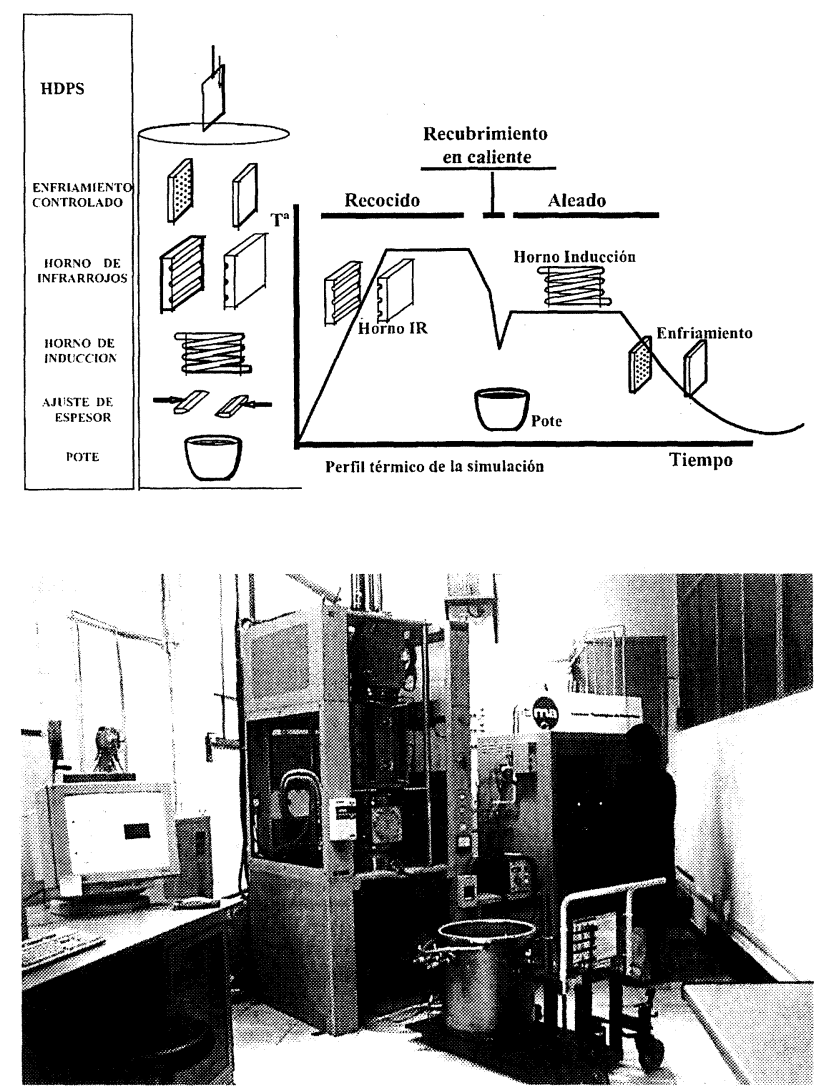

FIG. 1.- a) Vista esquemática del simulador de galvanizado en continuo y b) Vista global del mismo.

FIG. 1.- a) Schematic layout of the continuous hotdip process simulator and b) A general view. 
durante el proceso. Los parámetros de operación de conjunto y el perfil térmico real se registran en archivos para su posterior estudio y validación. La tabla I recoge las posibilidades de variación de los parámetros más importantes.

\section{RESULTADOS INICIALES}

Las muestras se han caracterizado de acuerdo con los siguientes ensayos:

- Peso de capa en caras separadas. Se evalúa gravimétricamente por decapado independiente de cada cara de la probeta con ácido clorhídrico 1:1 inhibido con hexametilentetramina de acuerdo con la norma UNE 36130-91 (EN 10 142). Los resultados se expresan en $\mathrm{g} / \mathrm{m}^{2}$ cara.

- Análisis químico del porcentaje de hierro. Sobre la disolución obtenida separadamente de cada cara en el ensayo anterior, se mide la concentración de hierro mediante ICP. El resultado se expresa como \% en peso de hierro en el recubrimiento de galvanorrecocido.

En primera aproximación se ajustaron los datos de peso de capa de chapas galvanizadas frente a los

TABLA I.- Posibilidades de variación de los parámetros de operación del simulador

TABLE I.- Operational variability of the hot-dip process simulator parameters

\begin{tabular}{|ll|}
\hline Recocido continuo & \\
Velocidad de calentamiento & $5-50{ }^{\circ} \mathrm{C} / \mathrm{s}$ \\
Velocidad de enfriamiento & $5-60{ }^{\circ} \mathrm{C} / \mathrm{s}$ \\
T ${ }^{\mathrm{a}}$ máxima de tratamiento & $950{ }^{\circ} \mathrm{C}$ \\
Atmósferas & \\
$\quad$ Inerte & Nitrógeno \\
$\quad$ Reductora & $0-25 \% \mathrm{H}_{2}$ \\
$\quad$ Punto de rocío & $-60 \mathrm{a}+5{ }^{\circ} \mathrm{C}$ \\
& \\
Recubierto & \\
Dimensiones de la probeta & $120 \times 200 \times 0,1-$ \\
& $3,2 \mathrm{~mm}$ \\
Espesor de recubrimiento & $40-300 \mathrm{~g} / \mathrm{m}^{2} \mathrm{cara}$ \\
Velocidad de la probeta & $10-1000 \mathrm{~mm} / \mathrm{s}$ \\
Control del espesor & $\mathrm{Cuchillas} \mathrm{de} \mathrm{N}_{2}$ \\
Aleación - horno inducción & \\
T ${ }^{\mathrm{a}}$ máxima de tratamiento & $760{ }^{\circ} \mathrm{C}(\mathrm{punto}$ Curie) \\
Velocidad de calentamiento & $0-150{ }^{\circ} \mathrm{C} / \mathrm{s}$ \\
Velocidad de la probeta & $200 \mathrm{~mm} / \mathrm{s}$ \\
Baño de metal fundido & \\
Temperatura máxima & $900{ }^{\circ} \mathrm{C} \pm 2{ }^{\circ} \mathrm{C}$ \\
Capacidad & $25-30 \mathrm{~kg}$ \\
\hline
\end{tabular}

parámetros de operación del simulador siguiendo un análisis dimensional. Esto permite definir la simulación cuando se desea, como objetivo, un determinado peso de capa en el galvanorrecocido.

Los parámetros mantenidos fijos fueron la temperatura del crisol, $460{ }^{\circ} \mathrm{C}$, la temperatura de entrada en el pote, $480{ }^{\circ} \mathrm{C}$ y el contenido de aluminio en el crisol, $0,12 \%$, variándose la velocidad de extracción de la chapa, $S \mathrm{w}$, la distancia de las boquillas de soplado para el control de espesor, $T$, y el flujo de nitrógeno de soplado, $F \mathrm{~W}$, siendo la variable de respuesta el peso de capa, $W$ (entre 40 y $300 \mathrm{~g} / \mathrm{m}^{2}$ ), obteniéndose:

$$
W=31,71(S \mathrm{~W})^{0.37} T^{0.83}(F \mathrm{~W})^{-0.30}
$$

con un coeficiente de correlación $R^{2}=0,935$ sobre 154 experimentos de simulación, siendo la desviación del peso de capa, para ensayos llevados a cabo en las mismas condiciones, del $6,2 \%$.

A partir de la ec. [1] se realizaron ensayos de galvanorrecocido con un peso de recubrimiento de $40 \mathrm{~g} / \mathrm{m}^{2}$, variándose el tiempo de aleación y la temperatura. Los resultados obtenidos muestran la cinética exponencial característica de estos recubiertos, como se aprecia en la figura 2.

En la figura 3 se han reunido las variaciones del contenido de hierro frente al contenido de fase $\delta$ del recubrimiento, obtenidas por electrodisolución anódica en régimen galvanostático con $1 \mathrm{~mA}$ de corriente y con el peso de recubrimiento, obteniéndose las dependencias habituales en estos recubrimientos.

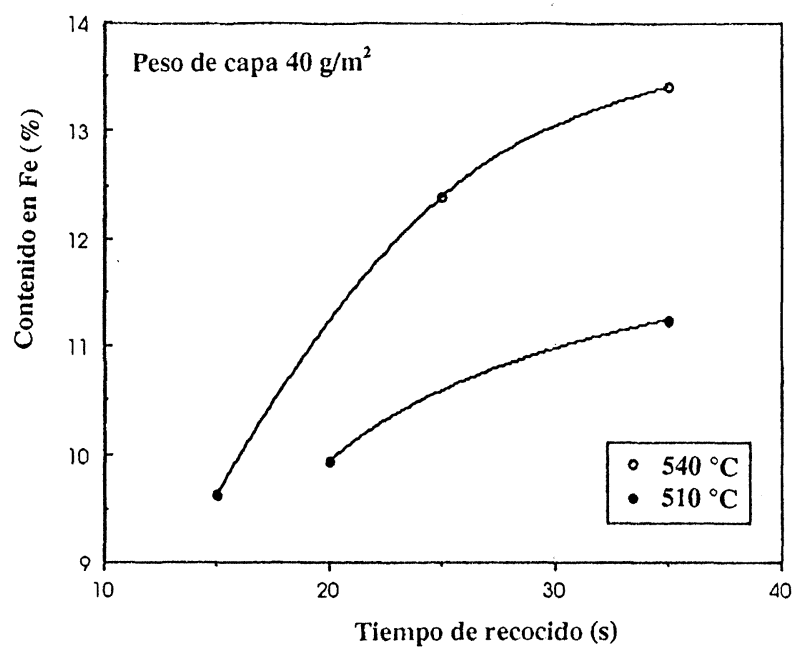

FIG. 2.- Contenido de hierro del galvanorrecocido frente al tiempo de recocido a 510 y $540^{\circ} \mathrm{C}$ (peso de recubrimiento $40 \mathrm{~g} / \mathrm{m}^{2}$ ).

FIG. 2.- Fe-content of galvanneal coating versus annealing hold time at 510 and $540{ }^{\circ} \mathrm{C}$ (coating weight $40 \mathrm{~g} / \mathrm{m}^{2}$ ). 

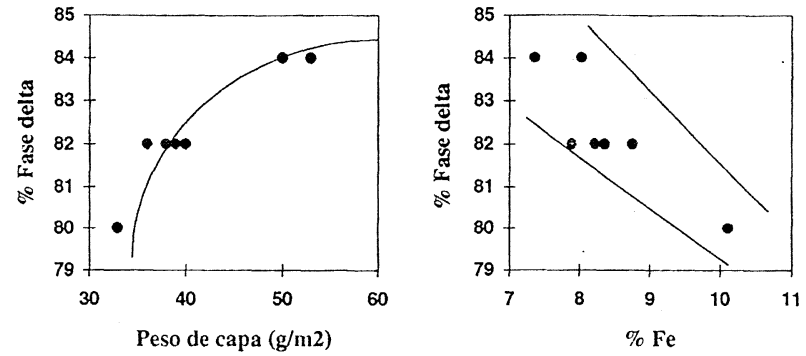

FIG. 3.- Contenido de fase $\delta$ del recubrimiento de galvanorrecocido frente al porcentaje de hierro y al peso de recubrimiento.

FIG. 3.- $\delta$ phase content of galvanneal coating versus \%iron content and coating weight.

\section{CONCLUSIONES}

La intrínseca complejidad de las reacciones que se establecen durante el aleado de un galvanizado para obtener un galvanorrecocido dentro del procedimiento industrialmente continuo de chapa es bien conocido por productores e investigadores. La falta de conocimiento de las condiciones reales que se establecen en las líneas continuas de tratamiento, junto con el trabajo en presencia de incertidumbre de los métodos discontinuos de laboratorio convencionalmente empleados para la generación de las fases aleadas y para su estudio, hacen extremadamente dificultosa su definición mecanicista y su expresión matemática cuantitativa.

La utilización de un simulador del proceso de galvanizado continuo y hornos de recocido para galvanorrecocidos similares a los utilizados en las líneas industriales permiten abordar el estudio de estos recubrimientos con garantía de proximidad a las condiciones industriales reales.

\section{REFERENCIAS}

(1) TANG, N-Y. y Adams, G.R. The Physical Metallurgy of Zinc Coated Steel. A.R. Marder Eds. Warrendale, Penn. (EE.UU.). 1993: 41-53.

(2) TAng, N.-Y. Metall. Mater. Trans. A, 26A, 1995: 16991704

(3) Jordan, C.E., Goggins, K.M., y Marder, A.R. Proc. 2nd Int. Conf. Zinc and Zinc Alloy Coated Steel Sheet. Galvatech'92. Amsterdam (Holanda). 1992: 137-141.

(4) Faderl, J., Pimminger, M. y Schönberger, L. Proc. Zinc and Zinc Alloy Coated Steel Sheet. Galvatech 92. Amsterdam (Holanda). 1992: 194-198.

(5) Kawaguchi, H. e Hirose, Y. The Physical Metallurgy of Zinc Coated Steel. A.R. Marder Eds. Warrendale, Penn. (EE.UU.). 1993: 153-167.

(6) Komatsu, A., Ando, A. y KittaKa, T. ISIJ Int. 37(3), 1997: 283-289

(7) Rensen, E. y van Eunsbergen, F.H. Galvanización, 22, 1993: 36-37

(8) Jordan, C.E. y Marder, A.R. The Physical Metallurgy of Zinc Coated Steel. A.R. Marder Eds. Warrendale, Penn. (EE.UU.). 1993: 197-219.

(9) Arai, M., Adachi, Y., Nakamori, T. y Usuki, N. ISIJ 83 (11), 1997: 713-718

(10) Mercer, P.D. Proc. Zinc and Zinc Alloy Coated Steel Sheet. Galvatech 92. Amsterdam (Holanda). 1992:204-207.

(11) Angeli, J., Faderl, J. y Gerdenitsch, J. The Physical Metallurgy of Zinc Coated Steel. A.R. Marder Ed. Warrendale, Penn. (EE.UU.). 1993: 221-240.

(12) Jenkins, G.A. y Burrows, L.J. Zinc and Zinc Alloy Coated Steel Sheet. Galvatech 92. Amsterdam (Holanda). 1992: 209-214.

(13) Zhang, L., Bensinger, T.R., Franke-Polz, E.M. y STRAYER, M.D. Zinc and Zinc Alloy Coated Steel Sheet. Galvatech 92. Amsterdam (Holanda). 1992: 215-219.

(14) LAMberigTs, M. y Leroy, V. Zinc and Zinc Alloy Coated Steel Sheet. Galvatech 92. Amsterdam (Holanda). 1992: 199-203.

(15) Burghardt, A.J.C., van der Heiden, A., van Perlstein, E.B. y Schoen, J.P. Zinc and Zinc Alloy Coated Steel Sheet. Galvatech 92. Amsterdam (Holanda). 1992: 189-193.

(16) Rios, P.R. Metall. Mater.Trans. A, 27A, 1996: 1.1321.134 . 